\title{
EchoGéo
}

\section{Les associations foncières pastorales : un dispositif de gouvernance concrète du foncier dans les territoires de montagne}

Laurence Barthe and Corinne Eychenne

\section{(2) OpenEdition \\ Journals}

Electronic version

URL: https://journals.openedition.org/echogeo/15228

DOI: 10.4000/echogeo.15228

ISSN: 1963-1197

Publisher

Pôle de recherche pour l'organisation et la diffusion de l'information géographique (CNRS UMR 8586)

Electronic reference

Laurence Barthe and Corinne Eychenne, "Les associations foncières pastorales : un dispositif de gouvernance concrète du foncier dans les territoires de montagne", EchoGéo [Online], 43 | 2018, Online since 22 March 2018, connection on 23 August 2021. URL: http://journals.openedition.org/echogeo/ 15228 ; DOI: https://doi.org/10.4000/echogeo.15228

This text was automatically generated on 23 August 2021.

EchoGéo est mis à disposition selon les termes de la licence Creative Commons Attribution - Pas d'Utilisation Commerciale - Pas de Modification 4.0 International (CC BY-NC-ND) 


\title{
Les associations foncières pastorales : un dispositif de gouvernance concrète du foncier dans les territoires de montagne
}

\author{
Laurence Barthe and Corinne Eychenne
}

Le recours au concept de gouvernance dans les travaux sur les politiques d'aménagement et de développement territorial a émergé dans un contexte très particulier marqué depuis les années 1980 par un processus de décentralisation, de territorialisation des politiques publiques et de multiplication de dispositifs visant à la co-construction de l'action publique. Ce concept permet d'analyser les différentes formes de coordination entre acteurs mais aussi entre échelles/niveaux (F-M Poupeau, 2017). Il permet de questionner l'évolution des formes du pouvoir et de sa distribution dans les territoires, dans une période où le pouvoir prescriptif de l'État reste fort mais davantage partagé avec l'Union européenne et les collectivités territoriales. La réussite de nombreuses politiques de développement territorial semble aujourd'hui associée à la capacité des territoires et de leurs acteurs à se coordonner, à construire de l'action collective autour d'enjeux de développement qui leur sont spécifiques. Les politiques en faveur des territoires de projet (parc naturel régional, pôle d'équilibre territorial rural, programme européen LEADER ${ }^{1}$ ), de la planification territoriale (Schéma de Cohérence Territoriale, Plan Local d'Urbanisme) ou de l'intercommunalité ont consacré de nouveaux cadres et échelles de gouvernance qui font l'objet de nombreux travaux depuis les années 2000, l'un des plus récents étant celui de Vanier et al. (2017) concernant les SCoT. Ces différents cadres ont donné lieu à l'affirmation de nouvelles pratiques de coopération et ont mis en avant de nouvelles figures de l'action publique locale tant politiques que techniques. Il existe cependant des dispositifs bien plus anciens intervenant à des échelles plus classiques du développement (commune) qui permettent d'observer des pratiques de gouvernance de proximité mobilisant des figures ordinaires de l'action publique locale. C'est à l'un de ces dispositifs, les associations foncières pastorales (AFP) que nous proposons de nous intéresser dans cet 
article. À partir d'une étude de terrain menée sur plusieurs AFP du massif pyrénéen, nous analysons une démarche concrète de gouvernance autour d'un objet singulier, le foncier, au cœur des préoccupations de gestion des acteurs du développement des territoires de montagne. Entre action prescriptive, fortement normée et descendante, et action volontariste d'élus locaux, de représentants de l'État, de techniciens du développement et de propriétaires fonciers, il s'agit d'identifier les mécanismes d'un processus de gouvernance discret et fonctionnel.

\section{Pour une approche située de la gouvernance territoriale : le cas des AFP}

2 L'étude de la gouvernance constitue un élément clef de la compréhension des démarches de développement local conduites dans les espaces ruraux depuis les années 1980. En effet, les modes de coopération et d'action collective entre différents acteurs sont devenus des caractéristiques intrinsèques de la conduite de stratégies, qu'elles soient sectorielles ou transversales, et qu'elles soient initiées par les politiques publiques d'appui au développement local ou directement par les acteurs locaux. Si la gouvernance ne constitue pas stricto sensu un seul et même idéal-type, elle n'existe pas non plus de facto. Elle caractérise un type de fonctionnement de l'action publique qui implique nécessairement une situation de coprésence entre des acteurs qui ont à gérer des questions collectives, mais qui se traduit au travers de diverses formes et est susceptible d'évoluer dans le temps. Elle renvoie à la coordination d'acteurs aux statuts divers, à l'organisation et l'agencement concertés de leurs interactions, pour prendre en charge une problématique commune, en vue d'établir un projet (Bertrand et al., 2004) ou plus simplement de conduire une action collective. "La gouvernance correspond à des modes de coordination associant aux acteurs publics (État, collectivités locales, etc.) des acteurs privés, qu'ils appartiennent au monde de l'entreprise (entreprises nationales ou transnationales) et/ou à la société civile comprise au sens large (associations, organisations non gouvernementales, syndicats, etc.), pour faire face à un problème complexe caractérisé par une multiplicité d'acteurs et d'échelles d'intervention» (Baron, 2003, p. 338). J.-P. Gilly et al. (2004) définissent pour leur part la gouvernance territoriale comme « un processus d'articulation dynamique de l'ensemble des pratiques et des dispositifs institutionnels entre des acteurs géographiquement proches en vue de résoudre un problème productif ou de réaliser un projet de développement ». Le concept de gouvernance territoriale permet ainsi d'analyser les mécanismes de mise en réseau de différents acteurs institutionnels, politiques, économiques et sociaux. Il invite à questionner les effets multiples de la proximité mis en avant par les politiques publiques ou activés par les acteurs locaux confrontés aux logiques de globalisation.

3 Au cours de nos travaux de recherche antérieurs, nous nous sommes particulièrement intéressées aux politiques de développement rural pour lesquelles la question de la gouvernance était à la fois une finalité et un moyen de mise en œuvre de ces politiques, l'instituant de fait comme une condition première de leur réussite et efficacité. Dans le cadre d'un programme Pour et Sur le Développement Régional (PSDR 3), Agriculture et Gouvernance des Territoires Ruraux, nous avons questionné les mécanismes de gouvernance à l'œuvre dans les territoires de projet (Parcs naturels régionaux, Pays au sens de la loi Voynet situés en montagne ou dans les espaces périurbains) en y 
observant plus spécifiquement la place de l'agriculture et des agriculteurs. Un constat majeur s'est dessiné révélant la difficile prise en charge des questions agricoles dans ces configurations de territoires de projet intervenant à des échelles intermédiaires (fédérations d'intercommunalités) face à la puissance des outils et acteurs sectoriels. Cependant, nous avons relevé l'existence de mécanismes de gouvernance partiels, où la place des agriculteurs et des organismes de développement agricole restait discrète. La «bonne gouvernance » recherchée par les territoires de projet apparaissait davantage dans les cadres de concertation proposés (comités de pilotage, conseils de développement, indiquant la présence de représentants du monde agricole) et dans les intentions affichées des projets que dans des actions concrètes, traduisant la difficile construction d'une action collective territorialisée autour de l'agriculture (Barthe et al., 2012 et 2013). Dans le cadre d'un programme de recherche en cours, $\mathrm{AFPYR}^{2}$, nous avons choisi de prolonger la question de la place de l'agriculture dans les dynamiques territoriales en privilégiant l'entrée foncière, abordée à travers un instrument puissant de restructuration foncière bien connu des services de développement en montagne mais singulièrement absent des travaux de recherche en sciences sociales : l'association foncière pastorale. Les AFP ont été créées par la loi pastorale de 1972 afin de résoudre les problèmes spécifiques $\mathrm{du}$ foncier en montagne: morcellement, indivisions, absentéisme des propriétaires, notamment sur les «zones intermédiaires " situées entre les fonds de vallée et les estives sur lesquelles les effets de la déprise agricole sont les plus prégnants (dynamique de végétation rapide, augmentation des risques naturels, proximité des villages et sentiment d'ensauvagement,...).

Les associations foncières pastorales ont pour objectif de regrouper les propriétaires de biens à vocation pastorale sur un périmètre défini collectivement au cours d'un processus de création qui repose sur une procédure strictement définie avec consultation écrite de tous les propriétaires par le préfet, enquête publique préalable et création par autorisation préfectorale au final ${ }^{3}$. Concrètement, la logique de création d'une AFP repose sur une règle de majorité et non d'unanimité (voir encadré de l'illustration 1), puisqu'elle permet d'intégrer au périmètre les parcelles appartenant à des propriétaires défavorables au projet. Ainsi, bien que dans le principe l'AFP ne touche pas au droit de propriété, il s'agit bien d'une possibilité de restriction, au nom de l'intérêt général, des droits de gestion et d'usage de la propriété privée (Comby, 2010). Les AFP apparaissent donc comme un levier puissant d'action sur le foncier, et conservent un potentiel d'innovation exemplaire, par leur dimension collective et contraignante sur les modalités d'usage et de gestion des biens privés. 
Illustration 1 - La règle de majorité dans la procédure de création d'une AFP autorisée (art. L13563 du Code rural)

Une AFP autorisée peut être constituée lorsqu'au moins $50 \%$ des propriétaires possédant au moins $50 \%$ de la surface des terres incluses dans le périmètre se sont prononcés favorablement. Dans le cas où une collectivité territoriale possède des terrains dans le périmètre, il n'est plus nécessaire d'avoir l'accord de plus de $50 \%$ des propriétaires, la majorité est atteinte lorsque les propriétaires possédant au moins $50 \%$ de la surface des terres incluses dans le périmètre se sont prononcés favorablement. Les propriétaires n'ayant pas répondu au courrier du préfet sont considérés comme favorables. Ceux donc la trace n'a pu être retrouvée (retour du courrier du préfet) ne sont pas comptabilisés. Au terme de la procédure, l'AFP est créée par autorisation préfectorale sur l'ensemble des parcelles du périmètre, y compris celles dont les propriétaires se sont prononcés défavorablement ${ }^{4}$.

Dans le cadre de cet article, nous faisons le choix d'interroger l'AFP en tant qu'elle constitue un cadre original d'analyse des mécanismes de gouvernance territoriale ${ }^{5}$, du fait de son caractère contraignant, fortement normé par le cadre juridique placé sous l'autorité du préfet, contrairement aux cadres de gouvernance souples et ouverts affichés dans les politiques de développement territorial comme les pôles d'équilibre territorial et rural (PETR) ou dans les parcs naturels régionaux (PNR) où il s'agit de susciter la participation des acteurs locaux dans leur diversité. En matière d'AFP, la gouvernance n'est pas affichée comme un principe préalable à l'action, mais sa nature, ses objectifs d'intervention, et ses modalités concrètes de mise en œuvre nous amènent à questionner les formes avérées (concrètes) de gouvernance territoriale déployées. L'objet même de l'AFP, qui vise à mettre en œuvre une gestion collective des terres face à une gestion privée jugée défaillante, instaure un contexte d'action sujet à tensions, le rapport à la propriété relevant d'une dimension individuelle très sensible. Ainsi, nous questionnons directement à travers la gestion du foncier le caractère potentiellement conflictuel de la gouvernance qu'aborde régulièrement André Torre dans ses travaux. «La gouvernance des territoires ne se limite donc pas à une vision idyllique des relations économiques et sociales, i.e. aux formes de coopération et de constructions communes » (Torre et Traversac, 2011). " Il s'agit également d'une interaction entre des forces poussant à la coopération et d'autres forces qui poussent au conflit » (Torre, 2011).

6 Nous pouvons ainsi formuler l'hypothèse que la création et le fonctionnement des AFP agissent sur les systèmes d'acteurs locaux pour constituer des espaces de gouvernance a priori contraints entre des acteurs aux ambitions contrastées voire contraires, qui permettent l'invention d'une action collective de proximité en faveur de l'agriculture. Nous partageons les approches de P-M Poupeau (2017) pour qui le concept de gouvernance apporte deux nouveaux éclairages sur la conduite de l'action publique que nous retrouvons dans le mode de mise en œuvre des AFP : en premier lieu, le retrait du rôle central de l'État qui reste certes un acteur de premier plan par la production règlementaire sur la création des AFP et son rôle de superviseur local de la procédure mais qui n'est pas « le protagoniste central de la régulation sociale ». En second lieu, la gouvernance des AFP révèle l'importance du rôle d'acteurs autres que les institutions locales : si leur fonction peut être décisive en matière d'impulsion d'un projet commun, elles ont besoin pour concrétiser leur projet de la mobilisation décisive d'autres acteurs, comme les propriétaires fonciers, les éleveurs locaux. 
7 L'analyse des mécanismes de gouvernance proposée ici s'inscrit dans une approche compréhensive fine de situations concrètes localisées socialement et spatialement. C'est la raison pour laquelle nous proposons dans la partie suivante de présenter les principaux éléments permettant d'appréhender les dynamiques à l'œuvre dans le cadre de la mise en œuvre d'associations foncières pastorales à travers trois études de cas de territoires situés sur le massif pyrénéen.

\section{La gouvernance en actes : une petite histoire des AFP sur trois territoires pyrénéens}

Dans le cadre du programme AFPYR, nous avons choisi d'amorcer un véritable travail d'exploration scientifique de l'objet AFP envisagé, au-delà de sa dimension instrumentale, comme un outil de négociation et de formalisation d'un projet territorial mettant en jeu différents systèmes de représentations et d'acteurs (élus, agriculteurs, propriétaires fonciers, techniciens) autour de questions relatives au rapport à la terre, à l'agriculture et à l'environnement en montagne. L'analyse fine des processus de création et de fonctionnement des AFP a été réalisée au travers de trois études de cas approfondies, situées dans les départements de l'Ariège (commune de Soulan dans le Couserans) et des Hautes-Pyrénées (vallée du Bastan en pays Toy et communes de Jézeau et Pailhac en moyenne vallée d'Aure)

Illustration 2 - Carte de localisation des AFP étudiées

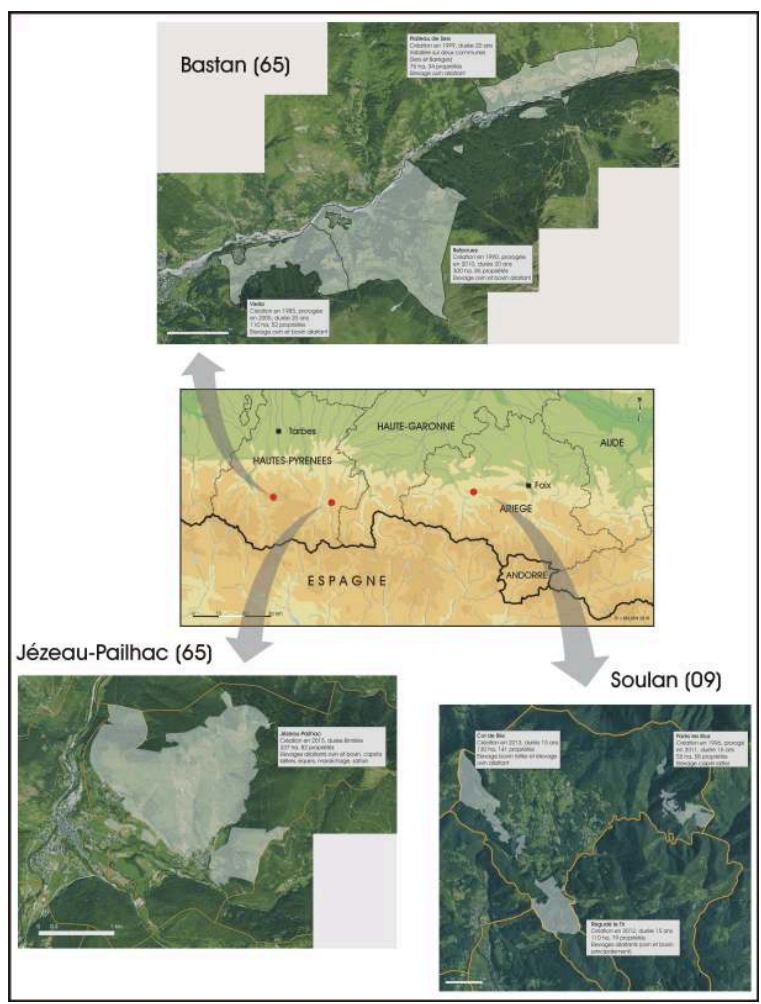

Ces trois territoires présentent des caractéristiques distinctes en matière de dynamiques de population, d'emploi ou d'évolution des milieux et des paysages. Ils sont concernés au total par 7 AFP aux profils variés quant à leur date de création, leur 
échelle d'action, les objectifs poursuivis, les acteurs impliqués, la nature des projets mis en œuvre, etc.

Illustration 3 - Les territoires d'étude

\begin{tabular}{|c|c|c|c|}
\hline & Soulan (09) & Vallée du Bastan (65) & \begin{tabular}{|l} 
Jézeau \& \& \\
Pailhac (65)
\end{tabular} \\
\hline & & $\begin{array}{l}\text { Communes de Barèges, Betpouey, } \\
\text { Esterre, Sers, Viella, Viey }\end{array}$ & \\
\hline Population 2013 & 357 & 689 & 168 \\
\hline $\begin{array}{l}\text { Évolution annuelle } \\
\text { 1968/1999 }\end{array}$ & $-0,70 \%$ & $-0,70 \%$ & $1,40 \%$ \\
\hline $\begin{array}{l}\text { Évolution annuelle } \\
1999 / 2013\end{array}$ & $0,70 \%$ & $-1,20 \%$ & $0,40 \%$ \\
\hline $\begin{array}{l}\text { Densité de la population } \\
2013\end{array}$ & 15 & 4,9 & 12,7 \\
\hline $\begin{array}{l}\text { Part des résidences } \\
\text { secondaires } 2013\end{array}$ & $59 \%$ & $83 \%$ & $39 \%$ \\
\hline $\begin{array}{l}\text { Emploi total au lieu de } \\
\text { travail } 2013\end{array}$ & 63 & 342 dont Barèges $78 \%$ & 20 \\
\hline $\begin{array}{l}\text { Nombre d'exploitations } \\
\text { agricoles } 2010\end{array}$ & 14 & 30 & 7 \\
\hline Évolution 1979/2000 & $-41 \%$ & $-29 \%$ & $0 \%$ \\
\hline Évolution 2000/2010 & $-30 \%$ & $-28 \%$ & $-56 \%$ \\
\hline SAU & 559 ha & $400 \mathrm{ha}$ & 80 ha \\
\hline Nombre d'AFP & 3 & 3 & 1 \\
\hline Surface totale en AFP & 300 ha & 490 ha & 340 ha \\
\hline Dates de création des AFP & $\begin{array}{l}1996-2012- \\
2013\end{array}$ & 1985-1990-1999 & 2015 \\
\hline $\begin{array}{l}\text { Nombre d'installations } \\
\text { liées aux AFP }\end{array}$ & 2 & & 4 \\
\hline
\end{tabular}




\begin{tabular}{|l|l|l|l|}
\hline & $\begin{array}{l}\text { ovins } \\
\text { allaitants } \\
\text { bovins } \\
\text { Types de production sur } \\
\text { les AFP }\end{array}$ & & \\
& $\begin{array}{l}\text { Maitants } \\
\text { bovins } \\
\text { laitiers } \\
\text { caprins } \\
\text { laitiers }\end{array}$ & $\begin{array}{l}\text { ovins allaitants } \\
\text { bovins allaitants }\end{array}$ & $\begin{array}{l}\text { caprins laitiers } \\
\text { ovins allaitants } \\
\text { équins }\end{array}$ \\
\hline
\end{tabular}

Sources : INSEE, Agreste, AFPYR. directifs réalisés auprès de 60 acteurs de ces territoires (propriétaires, agriculteurs, élus, techniciens), complétés par des temps d'immersion et d'observation prolongés sur le terrain, notamment lors des temps forts de la procédure et de l'action collective (assemblées générales, permanences du commissaire enquêteur, etc.) ainsi que par la consultation et l'analyse des archives des AFP étudiées.

11 Cette méthode nous permet de proposer une analyse de la "gouvernance ordinaire » d'une démarche de développement local discrète à plusieurs titres :

- parce qu'elle se déploie sur des territoires en marge des grandes logiques de métropolisation et de périurbanisation qui sont au cœur de la plupart des travaux actuels sur la gouvernance foncière (Gueringer et al., 2016) ;

- parce qu'elle met en scène des acteurs eux-mêmes " ordinaires » : élus de petites communes de montagne, agriculteurs, animateurs pastoraux et surtout micro-propriétaires fonciers habituellement invisibles ${ }^{6}$;

- parce qu'elle est associée à un « vieil » instrument d'action publique qui, lorsqu'il est connu, tend à être davantage associé à des formes de gouvernement autoritaires et descendantes qu'à une gouvernance ouverte et partenariale.

Il nous semble cependant que la petite histoire de vie des AFP permet d'éclairer différentes dimensions de la gouvernance territoriale relatives notamment à la mobilisation et la coordination des acteurs, aux échelles et aux modalités d'intervention, que nous mettrons en perspective dans une dernière partie avec certains travaux récents de portée plus générale consacrés à la question.

\section{Comment naissent les AFP?}

Les AFP, en tant qu'instrument d'action publique, ont été créées en 1972 pour répondre, via la structuration foncière, à des dynamiques d'évolution paysagère clairement identifiées et présentes sur la plus grande partie des espaces montagnards: embroussaillement et fermeture paysagère avérés ou redoutés du fait du recul plus ou moins ancien ou à prévoir de l'activité agricole et des modifications de pratiques vers l'agrandissement et la mécanisation. Ces dynamiques n'ont pas surgi soudainement, elles sont le fruit d'un processus inscrit dans la durée, prévisible et généralisé à l'échelle du massif. La nécessité de lutter contre ces évolutions jugées régressives n'est pas non plus une idée nouvelle, elle résulte d'un paradigme ancien, socialement construit et largement dominant, privilégiant espaces ouverts et présence agricole sur les territoires montagnards ${ }^{7}$. Tout se passe cependant comme si la «banalité » même 
du problème, et son ancienneté, représentaient autant de freins à l'action, comme une fatalité liée aux évolutions des sociétés montagnardes, qui explique en partie ${ }^{8}$ que l'ensemble du massif pyrénéen ne soit pas recouvert d'AFP, alors que l'efficacité du dispositif y est largement reconnue.

Dans les faits, la création d'une AFP est toujours issue d'un processus local, reposant sur la rencontre entre un problème converti en projet, une volonté portée par une figure locale et une ingénierie spécialisée.

Le rôle de l'élu local, en particulier communal, est central dans le portage des projets d'AFP. Par effet mécanique, puisque le poids des terrains communaux est généralement plus fort que celui des terrains privés dans la prise de décision (voir supra). Mais également et surtout parce qu'il bénéficie d'une forte reconnaissance locale de son pouvoir d'agir, du fait de son mandat qui lui garantit une légitimité institutionnelle, mais également de son ancrage dans le territoire et/ou l'activité agricole. Ainsi, les AFP de Soulan ont-elles été initiées par l'adjoint en charge des affaires agricoles, issu de la commune, fonctionnaire à la retraite, fils d'agriculteur qui aide son neveu repreneur de la ferme familiale. À Jézeau-Pailhac, l'AFP a été portée par les maires des deux communes, l'une agricultrice en activité, l'autre fils d'agriculteur. Et dans le Bastan l'origine des AFP doit être portée au crédit du maire de Betpouey, agriculteur pluriactif et figure également de la profession agricole dans les Hautes-Pyrénées.

L'étincelle qui va pousser, à un moment donné, ces figures locales à s'engager dans une démarche de création d'AFP peut être de nature diverse :

- demandes d'agriculteurs en place pour l'amélioration de leurs structures (restructuration foncière) ou de leurs conditions d'exploitations (équipements) : Bastan, AFP Parès à Soulan ;

- demandes de porteurs de projets agricoles pour obtenir des surfaces d'exploitation (JézeauPailhac, AFP Regudé et Col de Bès à Soulan) ;

- diminution rapide du nombre d'exploitations (Jézeau-Pailhac) ;

- effet de diffusion : rôle pédagogique des AFP déjà existantes sur le territoire ou sur des communes voisines (Soulan, Bastan, Jézeau-Pailhac) ;

- stratégie intercommunale voire départementale permettant de mettre à disposition de l'ingénierie spécialisée (politique volontariste ancienne du conseil départemental de l'Ariège en matière d'AFP, plus récente dans les Hautes-Pyrénées, utilisation d'une partie des fonds du plan de revitalisation du Couserans en Ariège mis en œuvre suite à la fermeture de l'usine Ledar, plan de gestion de l'espace intercommunal sur le canton d'Arreau,...).

\section{Le processus de création}

17 La création d'une AFP, comme toute démarche d'aménagement liée au foncier devant aboutir à la construction d'un compromis, est un processus long (deux à trois ans minimum) qui associe des dimensions procédurales et des phases d'animation. La mise en œuvre de la procédure nécessite la mobilisation conjointe d'acteurs très diversifiés : élus locaux, animateurs fonciers pastoraux, commissaire enquêteur, préfet.

La phase d'avant-projet vise à proposer un périmètre pour l'AFP. Il s'agit alors de réaliser un travail d'animation et de consultation auprès des acteurs locaux : éleveurs, propriétaires résidant ou non sur la commune, élus, chasseurs, techniciens agricoles et/ou forestiers. Cette étape articule réunions de groupes de travail autour du projet agricole et foncier, réunions d'information et visites individuelles, principalement auprès des propriétaires et des agriculteurs. Les animateurs fonciers des services 
pastoraux ${ }^{9}$ jouent un rôle central tout au long de la démarche. Dans les départements étudiés, chaque service pastoral dispose de deux animateurs spécialisés, véritables spécialistes des AFP, dont l'expertise est mise au service du porteur de projet pour mener à bien le travail de diagnostic et d'animation, la recherche de tous les propriétaires du périmètre pressenti ${ }^{10}$, et un travail de secrétariat (préfiguration des statuts de l'AFP, courriers aux propriétaires,...).

Pendant la phase d'avant-projet, les élus communaux sont fréquemment interpellés par des propriétaires/habitants ayant été informés de la démarche. En effet, la définition du périmètre est une étape hautement stratégique qui doit absolument bénéficier de la caution morale de l'élu. Ce qui se joue alors pour les propriétaires c'est la remise en cause de leur rapport à la propriété et de leur droit d'en décider l'usage, y compris pour eux-mêmes. L'ensemble du conseil municipal s'engage à la fin de cette phase d'avantprojet, via une délibération par laquelle la commune apporte à l'AFP l'ensemble des terrains communaux compris dans le périmètre. Durant toute la phase d'avant-projet, la dynamique est entièrement incarnée par le couple élu-animateur pastoral ${ }^{11}$, tous deux clairement et personnellement identifiés (les animateurs pastoraux sont toujours connus par leurs prénoms, leur appartenance institutionnelle est souvent plus floue pour les acteurs rencontrés). La réussite du processus repose non seulement sur leurs expertises conjointes (expertise, mandat, ancrage) mais également sur leurs qualités personnelles d'écoute, de respect, d'animation, de persuasion et de décision.

La procédure réglementaire peut alors être engagée, à travers un arrêté préfectoral de projet de création d'AFP qui ouvre l'enquête publique et lance la consultation par écrit de tous les propriétaires. Le choix du commissaire enquêteur est également stratégique. La spécificité de la procédure, sur un sujet assez technique aux enjeux très locaux, pousse à une forme de spécialisation des commissaires enquêteurs "AFP " généralement intéressés par les questions rurales et plutôt convaincus de l'utilité de l'outil. Généralement, le commissaire enquêteur n'est que peu sollicité par les propriétaires pendant l'enquête, qui conduit cependant souvent à des propositions de modifications mineures de périmètre, généralement à proximité des habitations. L'assemblée générale constitutive conclut la phase de création de l'AFP par décompte des voix des propriétaires et vérification des conditions de majorité. L'AFP est créée par arrêté préfectoral pour une durée variable. Elle élit ensuite en son sein un syndicat composé exclusivement de propriétaires, et un président (généralement un élu, sinon un agriculteur-propriétaire). Le syndicat réunit généralement les propriétaires les plus engagés dans la démarche, qu'ils y aient été ou non favorables. Il s'agit à la fois de "garder la main » sur la gouvernance de l'outil, et d'asseoir sa place dans le système d'acteurs local.

21 Au niveau communal, la création d'une AFP représente un temps fort de l'action collective car elle oblige chacun (à condition qu'il soit propriétaire) à prendre parti dans un projet global relatif à la gestion de l'espace et à la place de l'agriculture dans le territoire. Loin des discours généraux souvent unanimes sur l'intérêt de maintenir une activité agricole en montagne, l'AFP rentre dans le sujet de façon très concrète, en percutant de plein fouet le rapport à la propriété. Or ce rapport est complexe, il associe des référents patrimoniaux, filiation et ancrage territorial, des éléments matériels relatifs à une certaine pratique de l'espace (agriculture, chasse, mais aussi isolement choisi, autosuffisance alimentaire), ainsi que des, choix personnels quant au rapport à la nature, à l'anthropisation, à l'aménagement. En les sommant de donner ou non leur 
accord à la création de l'AFP, et par incidence au projet agricole associé, la procédure fait sortir de l'ombre des acteurs ordinaires généralement invisibles dans les démarches de développement local : les petits propriétaires fonciers de surfaces sans grande valeur monétaire. Ils se trouvent donc de fait associés à la validation, ou non, d'un projet territorial pour la commune et sont confrontés à l'exploration de leur propre rapport à la propriété, souvent implicite et intime. Sans qu'ils l'aient euxmêmes recherché, le projet de création de l'AFP leur ouvre une place dans une sphère collective pour eux inédite, dans laquelle ils se trouvent en situation de confrontation et d'échange avec d'autres propriétaires, des élus locaux, des techniciens. Le caractère normé de l'instrument AFP, qui oblige, et son objet, le foncier, qui implique, ouvrent donc de fait une arène de gouvernance locale originale qui ne cherche pas à éviter le conflit mais plutôt à le résoudre. En effet, la création d'une AFP ne se fait jamais sans heurts : en touchant à la propriété et au projet de territoire, elle met au jour voire génère des conflits parfois intenses et les assemblées générales peuvent être le théâtre de violentes altercations, qui trouvent parfois leur source bien ailleurs dans l'histoire des villages, des familles, et des personnes. Ces conflits ne sont pas esquivés, et la procédure ne prétend pas parvenir au consensus, puisque la règle de majorité s'impose à tous. Le caractère autoritaire de la procédure est cependant toujours atténué, dans les cas étudiés, par la reconnaissance de la qualité du travail d'animation effectué en amont, dans un contexte de proximité et d'interconnaissance fortes.

\section{Et après ?}

Après le temps chaud de sa création, l'AFP rentre dans une phase de fonctionnement: les terrains sont répartis entre les agriculteurs conformément au projet élaboré pendant la phase de création, des travaux pastoraux bénéficiant de financements publics peuvent être engagés (clôtures, débroussaillements, adductions d'eau, pistes...), qui peuvent encore faire l'objet de contestations quant au modèle d'aménagement de l'espace qu'ils promeuvent (Soulan), mais assez vite la fièvre retombe et les AFP rentrent dans une routine de fonctionnement, surtout si tout se passe bien au niveau des exploitations agricoles. Le syndicat assure la gestion quotidienne. Les animateurs pastoraux, toujours en lien étroit avec le président d'AFP, assurent le secrétariat et le suivi des AFP: convocation aux assemblées générales, montage de dossiers de financement,... jusqu'à ce que l'AFP atteigne sa date de prorogation qui nécessite de faire de nouveau voter les propriétaires, y compris bien sûr les conseils municipaux dont les membres et la stratégie peuvent avoir changé depuis la date de création. À ce jour, dans les départements étudiés, toutes les AFP ont été prolongées, ne serait-ce que parce que les conséquences de leur disparition en place seraient trop lourdes pour les exploitations agricoles. Mais le renouvellement ne se fait pas toujours sans heurts : les assemblées générales de prorogation peuvent faire de nouveau l'objet d'échanges violents, et la question de l'AFP est parfois là encore un argument de poids dans les joutes électorales pour les élections municipales.

\section{Expertise, légitimité, proximité, les ressorts d'une gouvernance locale fonctionnelle}

23 Alors que l'AFP pourrait apparaître comme un outil de gestion foncière un peu anachronique, du fait de son cadre procédural très éloigné des logiques fluides de la 
«bonne » gouvernance locale, l'analyse fine de ses modalités de mise en œuvre a mis au jour un cadre original, qui permet de proposer un certain nombre de déclinaisons spécifiques de quelques grandes catégories d'analyse de la gouvernance locale. Comme le précise Vincent Simoulin (2007), « le concept de gouvernance permet de réinterroger les concepts d'autorité et de légitimité dans un contexte de crise de l'action publique, d'hybridité croissante du public et du privé, de multiplication des dispositifs de "gouvernance instrumentale" ».

La mise en œuvre de l'AFP révèle en premier lieu l'existence d'un instrument d'action publique parfaitement normé avec ses outils, ses méthodes d'animation et de contrôle. L'hypothèse principale de la sociologie de l'action publique (Lascoumes et Le Galès, 2007) est que « la création d'instruments d'action publique peut servir de révélateur de transformations plus profondes de l'action publique, de son sens, de son cadre cognitif et normatif et des résultats". Dans le cas des AFP, le respect de la procédure règlementaire de création avec ses étapes obligatoires, ses acteurs de référence (élus communaux, propriétaires fonciers, préfet, commissaire enquêteur) et la mobilisation d'une ingénierie d'accompagnement de cette démarche (animateurs pastoraux) donnent lieu à une configuration originale où l'expertise règlementaire et administrative est en interaction avec une expertise technique (animation de projet) et une expertise d'usage (celle des élus, des propriétaires, des éleveurs). La procédure règlementaire devient chemin faisant le cadre d'un débat souvent controversé autour d'un projet de gestion du territoire et de développement de l'agriculture. Si parfois les situations de gouvernance donnent lieu à des formes de domination ou d'accaparement des démarches par certains élus et techniciens (Simoulin, 2007), la création des AFP avec ses règles procédurales oblige à un certain partage du pouvoir d'expertise car tous les protagonistes, du fait de l'objet d'action, le foncier, ont un pouvoir d'influence voire de blocage réel. L'équilibre de cette configuration est étroitement lié aux conditions de légitimité dont disposent les acteurs ou qu'ils construisent pour stabiliser leurs rôles.

La mise en œuvre et le fonctionnement de l'AFP mettent en effet en exergue l'importance des formes classiques de légitimité de certaines figures de l'action publique locale, aujourd'hui un peu oubliées ou délaissées par les recherches actuelles portant sur le développement territorial. Les maires, voire certains conseillers municipaux, jouent un rôle déterminant dans la démarche de coordination et d'engagement de tous les protagonistes, éleveurs comme propriétaires. Le statut de l'élu local, son pouvoir d'agir sur le territoire sont largement reconnus par tous les acteurs et renforcent son pouvoir de persuasion. Dans le cas des AFP, cette légitimité est renforcée par la capacité de l'élu à inscrire son action dans le temps long de l'histoire du territoire et de ses habitants. La mobilisation récurrente de la propriété communale, caractéristique forte des usages de l'espace en montagne, dans le projet de création de l'AFP permet de mettre en avant le registre de la tradition: l'héritage commun est mis au service du projet de territoire et il convoque en écho les héritages des autres propriétaires privés, et le souvenir du caractère patrimonial du paysage agricole ouvert, issu du labeur des générations précédentes. La dimension personnelle de la figure de l'élu joue également un rôle essentiel dans l'acceptabilité du projet, notamment pour les propriétaires endogènes : sa trajectoire familiale, professionnelle, son ancrage local à travers sa forte reconnaissance par les habitants anciens ou plus récents, sa capacité de conviction et de médiation, ses différentes implications participent de la constitution d'une figure charismatique (Barthe et Eychenne, 2014) 
qui pèse activement dans le processus d'organisation de l'AFP. Le maire, en mobilisant ces différentes ressources, dispose d'un capital de confiance et, en tant que porteur du projet d'AFP, il les mobilise activement et de manière différenciée pour convaincre. Ces différentes formes de légitimité sont mobilisées au service de l'action locale : l'élu agit pour traiter des problèmes qui affectent la collectivité.

Comme l'indique Duran (2009): "Si le politique est toujours la manifestation de rapports de forces, de jeux de pouvoir et de stratégies de domination, il est aussi une activité de fixation des buts collectifs qui se cristallisent dans l'énoncé et la mise en œuvre de politiques publiques, c'est-à-dire dans la capacité à définir des buts collectifs, à mobiliser les ressources nécessaires à leur poursuite, à prendre les décisions qu'impose leur obtention et à assumer au final les conséquences qui en découlent ». L'élu joue de sa légitimité pour agir, modifier un ordre établi qui peut soit conforter sa position, soit la fragiliser. Même si, lorsqu'il engage la procédure de création d'une AFP, il est à peu près sûr de voir la démarche aboutir, il prend ouvertement le risque du conflit, des controverses entre propriétaires, éleveurs, population locale et de l'absence d'engagement collectif. Il prend également un risque politique plusieurs fois vérifié, l'AFP pouvant être une ligne de fracture centrale lors des élections municipales. Sa légitimité pour se perpétuer a besoin de l'engagement des autres parties prenantes, un des enjeux étant souvent de confier après sa création le portage de l'AFP à des éleveurs.

L'affirmation de la légitimité de l'élu communal comme du technicien pastoral fait apparaître une autre caractéristique des formes de gouvernance mises en œuvre à travers les AFP. Elle concerne l'importance de la proximité. Il s'agit certes d'une caractéristique intrinsèque des formes de gouvernance territoriale inscrites dans des territoires de proximité mais elle prend dans notre cas d'étude un contour particulier puisqu'ici elle s'exprime à une échelle aujourd'hui bousculée de l'organisation territoriale, celle de la commune, qui se voit doublée par l'échelle intercommunale qui acquiert de plus en plus de compétences en matière de développement territorial. La proximité maintes fois définie depuis les années 1990 dans le cadre des recherches du groupe Dynamiques de proximité (Torre 2010, 2011; Bouba-Olga et Grossetti, 2008) permet d'identifier deux dimensions caractéristiques de ce concept. La proximité géographique permet d'appréhender la distance spatiale entre des personnes ou entre des personnes et des éléments matériels (un paysage, une entreprise) ; elle repose aussi sur des éléments subjectifs à travers la perception de cette distance et elle dépend de facteurs techniques comme les aménagements qui permettent une capacité plus ou moins facilitée d'accès, de déplacement. La proximité organisée se construit, quant à elle, à travers les différentes manières qu'ont des acteurs d'interagir à travers différents cadres ou organisations d'autre part. Nous privilégions l'approche proposée par Bouba-Olga et Grossetti (2008) qui proposent une définition de la proximité organisée autour de deux composantes : la proximité de ressources qui peut relever de ressources cognitives ou matérielles (patrimoine, revenus, niveaux de formation) et la proximité de coordination qui s'appuie sur l'activation de réseaux sociaux et sur le rôle de médiation que peuvent jouer des dispositifs facilitant ou imposant des échanges. Dans le cas de l'analyse des formes de gouvernance présentes dans les AFP, le rôle de la proximité géographique s'avère déterminant pour le succès de la démarche souvent confrontée à des logiques conflictuelles : la référence à l'identité communale dans des territoires de montagne souvent caractérisés par l'isolement et l'éloignement des centres, à l'attachement au territoire de résidence ou d'origine, aux caractéristiques physiques de l'espace, constituent des activateurs de mobilisation et d'implication tant 
pour les élus que pour les propriétaires fonciers. De même, le recours fréquent aux rencontres en face à face, y compris sur les lieux concernés par le projet de réorganisation foncière, ainsi que les formes d'observation directe et fréquente $d u$ paysage environnant et/ou des activités des voisins agriculteurs, témoignent des effets accélérateurs et pragmatiques de la proximité géographique dans la construction d'une représentation de l'espace. L'effet de proximité géographique augmente les dynamiques liées à la proximité organisée : le partage de valeurs communes autour du développement local, du travail de gestion et d'entretien des espaces par l'agriculture, l'appartenance au groupe des propriétaires fonciers et plus globalement l'appartenance à la communauté villageoise revendiquée ou subie sont autant de composantes de la proximité de ressources. Enfin, la proximité de coordination imposée par le cadre AFP active la situation d'action collective en remobilisant les acquis de la proximité géographique et de la proximité de ressources, en les mettant en scène à travers discours et projets pour le territoire. L'AFP est un véritable dispositif de médiation locale : sa concrétisation ne peut se faire sans échanges, sans décision collective entre des acteurs qui s'inscrivent résolument dans une action concrète de proximité.

\section{Conclusion}

L'analyse des modes de gouvernance mis en œuvre autour de la création et du fonctionnement des AFP permet de mettre au jour des dynamiques concrètes de coordination d'acteurs a priori fort éloignés de l'action publique locale. Loin des dispositifs actuels qui font de la gouvernance ouverte un principe préalable à l'action, les AFP tranchent dans le paysage du développement rural du début du $\mathrm{xxI}^{\mathrm{e}}{ }^{\circ}$ siècle par le caractère normé, voire autoritaire, d'une procédure inventée il y a 45 ans, dans un tout autre contexte de référentiel d'action publique. Et pourtant, elles permettent la mise en mouvement de formes d'action collective localisées extrêmement originales grâce à trois éléments majeurs : la procédure (réglementaire) qui oblige, l'objet (le foncier) qui implique et l'échelle (la commune) qui facilite. La proximité sociale et géographique est l'ingrédient central qui permet d'expliquer la réussite des AFP. Ici, chacun est socialement et individuellement identifié, les notions d'ancrage, de confiance, d'interconnaissance sont au cœur des dynamiques collectives, qu'elles relèvent de la coordination ou du conflit. Cependant, au-delà de la «simple » affaire municipale, l'AFP porte un projet de territoire sur lequel qui s'abstient consent. En touchant au principe même de la liberté individuelle le plus souvent associé à l'idée de propriété privée, l'AFP oblige donc à l'engagement dans l'action collective, elle ne cherche pas à éviter le conflit, omniprésent, mais à le résoudre dans la construction d'un compromis pouvant servir de base à l'élaboration d'un projet territorial, si ce n'est partagé par tous, au moins discuté et donnant lieu à des transformations avérées du territoire de ses activités. 


\section{BIBLIOGRAPHY}

Baron C., 2003. La gouvernance : débats autour d'un concept polysémique. Droit et société $/ 2, \mathrm{n}^{\circ} 54$, p. 329-349.

Barthe L., Eychenne C., 2014. Agriculture et développement des territoires ruraux : des légitimités d'action en contradiction. In Sarrazin F. (dir.), Les élites agricoles et rurales. Presses universitaires de Rennes, p. 389-404.

Barthe L., Duvernoy I., Eychenne C. et Milian J., 2012. Agriculture et développement territorial. Sud-Ouest Européen, n 34, 92 p.

Barthe L., Duvernoy I., Eychenne C. et Milian J., 2013. Agriculture et développement territorial (partie II). Sud-Ouest Européen, $\mathrm{n}^{\circ}$ 35, 96 p.

Bertrand N., Moquay P., 2004. La gouvernance locale, un retour à la proximité. Économie Rurale, $\mathrm{n}^{\circ} 280$, p. $77-94$.

Bouba-Olga O., Grossetti M., 2008. Socio-économie de proximité. Revue d'Économie Régionale \& Urbaine, $\mathrm{n}^{\circ}$ 3, p. 311-328.

Comby J., 2010. Territoire commun et propriétés privées. Études foncières, n 143, p. 23-25.

Duran P., 2009. Légitimité, droit et action publique. L'Année sociologique, vol. 59, p. 303-344.

Gilly J.-P., Leroux I., Wallet F., 2004. Gouvernance et proximité. In Pecqueur B., Zimmermann J.-B., Économie de proximités. Hermès Science Publications, p. 187-206.

Lascoumes P., Le Gales P., 2007. Sociologie de l'action publique. Armand Colin, 126 p.

Poupeau F.-M., 2017. Analyser la gouvernance mutli-niveaux. Presses universitaires de Grenoble, Collection Politique en plus, 263 p.

Schnitzler A., Genot J.-C., 2012. La France des friches, de la ruralité à la féralité. Ed. Quae, 186 p.

Simoulin V., 2007. La gouvernance territoriale : dynamiques discursives, stratégiques et organisationnelles. Droit et Société, vol. 44, p. 15-32.

Torre A., 2010. Jalons pour une analyse dynamique des Proximités. Revue d'Économie Régionale \& Urbaine, $\mathrm{n}^{\circ}$ 3, p. 409-437.

Torre A., 2011. Les processus de gouvernance territoriale. L'apport des proximités. Pour, $\mathrm{n}^{\circ}$ 209-210, p. 114-122.

Torre A., Traversac J. B. 2011. Territorial Governance. Local Development, Rural Areas and Agrofood Systems. New York, Springer Verlag, 207 p.

Vanier M., Faure A., Dugua B., Loisel M., Martin-Gousset C., 2017. La planification à la croisée des chemins : les SCoT comme cas d'école. Pouvoirs Locaux : les cahiers de la décentralisation, Institut de la décentralisation, p. 33-38.

\section{NOTES}

1. Programme de Liaison entre les Actions de Développement de l'Économie Rurale, programme d'appui au développement local rural lancé par l'UE en 1991, aujourd'hui composante de la politique européenne de développement rural. 
2. «Les associations foncières pastorales dans les Pyrénées : mobilisation foncière et projet de territoire " (AFPYR). Programme financé par la Région Occitanie, qui regroupe une équipe pluridisciplinaire de 10 chercheurs (géographie, aménagement, paysage, sociologie) de l'UMR LISST-Dynamiques rurales (UT2J et ENSFEA) et de l'UMR Dynafor (INRA).

3. La loi prévoit trois types d'AFP, mais la plus répandue sur le massif pyrénéen, comme sur l'ensemble du territoire national, est l'AFP autorisée sur laquelle nous avons centré nos travaux.

4. Les dispositions législatives relatives aux AFP figurent dans le Code rural, Livre ${ }^{\mathrm{er}}$, titre III, chapitre V - Les associations foncières pastorales, art. L135-1 à L135-12.

5. L'équipe du programme AFPYR a publié ou soumis par ailleurs des analyses relatives à d'autres axes de questionnement: rapport au paysage (revue Projets de paysage), à la propriété et au modèle agricole (colloque La renaissance rurale, d'un siècle à l'autre, Toulouse, 2016; revue Métropoles). Une exposition de synthèse a également été réalisée, consultable sur le blog du programme : https://afpyr.hypotheses.org/

6. Ces propriétaires fonciers ont un lien très variable à la commune (et à leur propre propriété) : certains y résident, d'autres n'y viennent que pour les vacances ou les week-ends, d'autres habitent très loin et n'ont parfois aucun lien avec la commune, ignorant qu'ils y sont propriétaires d'une parcelle du fait d'un lointain héritage...

7. Pour approfondir la question de la construction sociale du rapport à l'enfrichement, voir Schnitzler et Génot, 2012.

8. Le risque politique lié à l'engagement en faveur des AFP, que nous exposerons par la suite, explique également la prudence voire l'opposition de certains élus.

9. Les services pastoraux (sur nos terrain : Fédération pastorale en Ariège et Centre de ressources sur le pastoralisme et la gestion de l'espace dans les Hautes-Pyrénées) ont été créés dans les années 1980-1990 afin de prendre le relais des services de l'état dans la mise en œuvre de la loi pastorale de 1972, notamment en accompagnant les dynamiques collectives liées aux groupements pastoraux et aux associations foncières pastorales. En 2017, l'Ariège compte $66 \mathrm{AFP}$, les Hautes-Pyrénées 17 AFP (292 AFP en France en 2011).

10. Nous ne détaillerons pas ici mais il s'agit d'un travail absolument titanesque. Pour les AFP étudiées, en moyenne 80 propriétaires pour 125 ha par AFP, dont de multiples toutes petites propriétés privées souvent en indivision et dont les propriétaires ne sont pas toujours connus au moment du lancement de la démarche.

11. Auquel s'ajoute le rôle de l'ombre absolument nécessaire du/de la secrétaire de mairie.

\section{ABSTRACTS}

The pastoral land associations are tools of land restructuring created by the pastoral law of 1972 to propose a solution to the specific problems of the land in mountain (division, joint possessions, absentee owners). It is a very standardized statutory instrument, which nevertheless allows to implement original forms of governance thanks to three major elements: the (statutory) procedure which obliges, the object (the land) which involves and the scale (the municipality) which facilitates. Thus social and geographical closeness appears as the central ingredient which allows to explain the success of AFP.

Les associations foncières pastorales sont des outils de restructuration foncière créés par la loi pastorale de 1972 pour proposer une solution aux problèmes spécifiques du foncier en montagne 
(morcellement, indivisions, propriétaires absentéistes). Il s'agit d'un instrument réglementaire très normé, qui pourtant permet de mettre en œuvre des formes de gouvernance originales grâce à trois éléments majeurs : la procédure (réglementaire) qui oblige, l'objet (le foncier) qui implique et l'échelle (la commune) qui facilite. La proximité sociale et géographique apparaît donc comme l'ingrédient central qui permet d'expliquer la réussite des AFP.

\section{INDEX}

Mots-clés: proximité, foncier, montagne, gouvernance, interconnaissance, instrument

Keywords: closeness, land, mountain, governance, interknowledge, instrument

Subjects: Sur le Champ - Sur le Terrain

\section{AUTHORS}

\section{LAURENCE BARTHE}

Laurence Barthe, barthe@univ-tlse2.fr, est Maître de conférences en géographie et aménagement, UMR LISST-Dynamiques rurales, Université Toulouse Jean Jaurès. Elle a publié récemment :

- Barthe L., Bustos-Cara R., Petrantonio M., Taulelle F., 2017. La municipalité argentine, acteur du développement local ? In Bonnal P., Chevalier P., Sourisseau J-M, Dedeire M. (dir.), Production et circulation des normes pour l'action territoriale. Presses universitaires de Méditerranée, Collection Territoires en mutation, p. 225-238.

- Barthe L., Eychenne C., 2014. Agriculture et développement des territoires ruraux : des légitimités d'action en contradiction. In Sarrazin F. (dir.), Les élites agricoles et rurales. Presses universitaires de Rennes, p. 389-404.

- Barthe L., 2014. Les territoires de proximité, creusets d'initiatives de développement ? In Dugot P., Thuillier G. (dir.), France : les mutations des systèmes productifs. PUM, p. 183-195.

\section{CORINNE EYCHENNE}

Corinne Eychenne, corinne.eychenne@univ-tlse2.fr, est Maître de conférences en géographie, UMR LISST-Dynamiques rurales, Université Toulouse Jean Jaurès.

- Eychenne C., Lazaro L., Gambino M., à paraître 2018. L'AFP ou la lutte des places au village, le cas de la commune de Soulan en Ariège. Communication au colloque La renaissance rurale d'un siècle à l'autre, Toulouse, à paraître aux PUM.

- Eychenne C., Lazaro L., 2014. L'estive entre « biens communs » et « biens collectifs ». Journal of Alpine Research / Revue de géographie alpine [En ligne], 102-2 | 2014, mis en ligne le 24 mars 2014. http://rga.revues.org/2297 ; DOI : 10.4000/rga.2297.

- Eychenne C., 2014. L'agriculture de montagne, de la marginalité au capital d'innovation. In Dugot P., Thuillier G. (dir.), France : les mutations des systèmes productifs, PUM, p. 383-397. 